Research Paper

\title{
Antiemetic Effectiveness and Cost-Saving of Aprepitant plus Granisetron Is Superior to Palonosetron in Gastrointestinal Cancer Patients Who Received Moderately Emetogenic Chemotherapy
}

Haruka Toda $^{1 *}$, Hitoshi Kawazoe ${ }^{* \star 凶}$, Akiko Yano $^{1}$, Yuji Yamamoto ${ }^{2}$, Yuji Watanabe ${ }^{2}$, Yasunori Yamamoto $^{3}$, Yoichi Hiasa ${ }^{4}$, Yoshihiro Yakushijin ${ }^{5}$, Akihiro Tanaka ${ }^{1}$, and Hiroaki Araki ${ }^{1}$

1. Division of Pharmacy, Ehime University Hospital, Shitsukawa, Toon, Ehime 791-0295, Japan;

2. Division of Gastrointestinal Surgery and Surgical Oncology, Department of Surgery, Ehime University Graduate School of Medicine, Shitsukawa, Toon, Ehime 791-0295, Japan;

3. Endoscopy Center, Ehime University Hospital, Shitsukawa, Toon, Ehime 791-0295, Japan;

4. Department of Gastroenterology and Metabology, Ehime University Graduate School of Medicine, Shitsukawa, Toon, Ehime, 791-0295, Japan;

5. Cancer Center, Ehime University Hospital, Shitsukawa, Toon, Ehime 791-0295, Japan.

* These authors contributed equally to this work.

$\triangle$ Corresponding author: Hitoshi Kawazoe, Division of Pharmacy, Ehime University Hospital, Shitsukawa, Toon, Ehime 791-0295, Japan. Phone: +81-89-960-5738; Fax: +81-89-960-5745; E-mail: hitoshik@m.ehime-u.ac.jp

(C) Ivyspring International Publisher. This is an open access article distributed under the terms of the Creative Commons Attribution (CC BY-NC) license (https://creativecommons.org/licenses/by-nc/4.0/). See http://ivyspring.com/terms for full terms and conditions.

Received: 2016.08.04; Accepted: 2017.04.08; Published: 2017.05.12

\begin{abstract}
Purpose The therapeutic benefit of a three-drug combination of antiemetics has not been established in moderately emetogenic chemotherapy (MEC). The aim of this study was to compare the antiemetic effectiveness and cost-saving of palonosetron plus dexamethasone (control group) with aprepitant, granisetron, and dexamethasone (study group) in cancer patients who received MEC.

Methods We switched the standard antiemetic treatment from the control group to the study group in gastrointestinal cancer patients who received MEC after October 2015. The antiemetics in both groups were modified using salvage antiemetic therapy at the clinicians' discretion, according to the severity of chemotherapy-induced nausea and vomiting. We retrospectively reviewed the electronic medical records from patients, before and after switching groups, from between April 2014 and March 2016.

Results We evaluated 443 treatment courses in 83 patients. The proportion of courses that included salvage antiemetic therapy in the control group and the study group was $34.8 \%(116 / 333)$ and $8.2 \%(9 / 110)$, respectively, and was statistically significant $(p<0.001)$. The mean integrated costs of antiemetics per course in the control group and the study group were $193 \pm 55$ USD and $143 \pm 38$ USD, respectively. Multivariate logistic regression analysis revealed that the study group was significantly associated with a reduced risk of requiring salvage antiemetic therapy $(p=0.038)$.

Conclusions These results suggest that the antiemetic effectiveness and cost-saving of a three-drug combination of aprepitant, generic granisetron, and dexamethasone was superior to a two-drug combination of palonosetron plus dexamethasone in gastrointestinal cancer patients who received MEC.
\end{abstract}

Key words: aprepitant; palonosetron; moderately emetogenic chemotherapy; gastrointestinal cancer; cost-saving.

\section{Introduction}

Gastrointestinal cancer, including colorectal and gastric cancer, is a major cause of morbidity and mortality globally. Oxaliplatin and irinotecan are key drugs for patients with gastrointestinal cancer [1-4], and are categorized as moderately emetogenic chemotherapy (MEC) according to several international and national antiemetic guidelines [5-8]. Chemotherapy-induced nausea and vomiting (CINV) 
can reduce patients' quality of life. Various guidelines recommend that the standard antiemetic treatment for CINV in patients receiving MEC is a two- or three-drug combination of a 5-hydroxytryptamine-3 receptor antagonist (5- $\left.\mathrm{HT}_{3} \mathrm{RA}\right)$ and dexamethasone, with or without a neurokinin 1 receptor antagonist ( $\mathrm{NK}_{1}$ RA) [5-8]. Palonosetron, a second-generation $5-\mathrm{HT}_{3} \mathrm{RA}$, is recommended for MEC as a preferred $5-\mathrm{HT}_{3} \mathrm{RA}$ in the National Comprehensive Cancer Network and American Society of Clinical Oncology antiemetic guidelines [6, 7]. The therapeutic benefit of a three-drug combination of a $\mathrm{NK}_{1} \mathrm{RA}, 5-\mathrm{HT}_{3} \mathrm{RA}$, and dexamethasone has not been established for MEC. However, a recent phase III trial showed that a three-drug combination was superior to a two-drug combination of a 5- $\mathrm{HT}_{3} \mathrm{RA}$ and dexamethasone for colorectal cancer patients who had received an oxaliplatin-based regimen. The number of patients who did not experience vomiting in the acute and delayed phases was statistically significant for the three-drug combination group [9]. In addition, a three-drug combination had almost comparable antiemetic efficacy between a first- and second-generation 5- $\mathrm{HT}_{3}$ RA [9]. Granisetron, a first-generation $5-\mathrm{HT}_{3} \mathrm{RA}$, is available as a generic drug in Japan. Population aging is progressing in Japan and other developed countries, resulting in increased health care costs. The difference in antiemetic efficacy and cost-saving between a twoand three-drug combination for MEC remains an unresolved issue within clinical practice. The aim of this study was therefore to compare the antiemetic effectiveness and economic impact of the recommended two-drug combination of palonosetron plus dexamethasone (control group) with a three-drug combination of aprepitant, generic granisetron, and dexamethasone (study group) in gastrointestinal cancer patients who received MEC.

Standard antiemetics

Control group (Before October 2015)

Two-drug combination

Palonosetron+dexamethasone

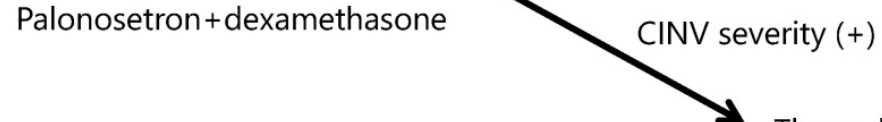

Three-drug combination

Aprepitant+palonosetron+dexamethasone

\section{Materials and Methods}

\section{Study design}

We switched the standard antiemetics from the control group to the study group in gastrointestinal cancer patients who received an oxaliplatin- or irinotecan-based regimen after October 2015. Patients in the control group received intravenous palonosetron $(0.75 \mathrm{mg})$ and generic dexamethasone $(6.6 \mathrm{mg})$ for 15 minutes immediately prior to chemotherapy on day 1, followed by oral dexamethasone $(8 \mathrm{mg})$ on days $2-3$. In contrast, patients in the study group received an oral aprepitant (125 mg) 60 minutes prior to chemotherapy plus intravenous generic granisetron $(1 \mathrm{mg})$ and generic dexamethasone ( 3.3 or $6.6 \mathrm{mg}$ ) for 15 minutes immediately prior to chemotherapy on day 1 , followed by oral aprepitant $(80 \mathrm{mg})$ and oral dexamethasone $(4 \mathrm{mg}$ ) on days $2-3$. The use of rescue medication, defined as any medication taken to treat CINV, was administered to each patient as needed and subsequent antiemetics in both groups were modified to salvage antiemetic therapy, using the best available three-drug combination of aprepitant, palonosetron, and dexamethasone at the clinicians' discretion, according to CINV severity. The salvage antiemetic therapy was also administered using the described process. The study design chart is shown in Fig. 1.

This retrospective observational study was carried out at Ehime University Hospital using data from electronic medical records dated between April 2014 and March 2016. We extracted the necessary clinical information about patient demographics, including age, sex, previous chemotherapy, chemotherapy regimen, chemotherapy dose, antiemetic use, CINV, and use of rescue medication, from colorectal or gastric cancer patients who had received at least two courses of MEC.

Salvage antiemetic therapy

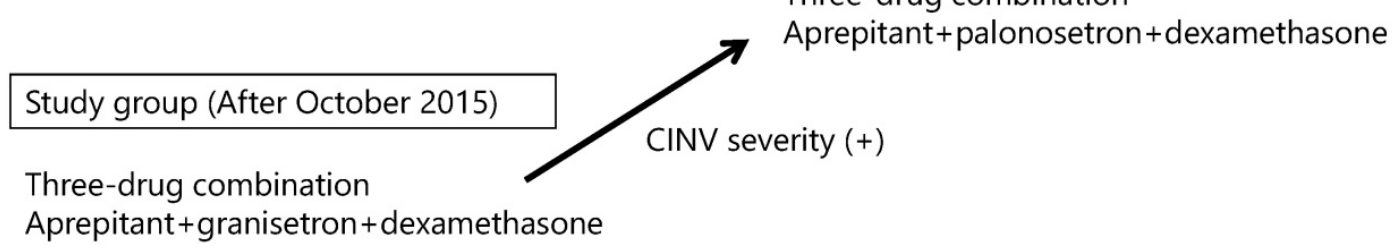

Figure 1. Study design chart. CINV: chemotherapy-induced nausea and vomiting 
Subjects meeting any of the following criteria were excluded from this study: 1) complications that induced nausea and/or emesis (e.g., symptomatic brain metastases, ulcerative diseases, and severe hepatic dysfunction); 2) administration of drugs which cause nausea and/or emesis during the investigation period, except for antiemetics (e.g., major or minor tranquilizers, or corticosteroids for any other reason); 3) concomitant radiotherapy at an esophageal site during the investigation period; and 4) administration of total parenteral nutrition.

The study protocol was approved by the ethics committee of Ehime University Hospital (approval number: 1606003) and was conducted in accordance with the Declaration of Helsinki and the Ethical Guidelines for Medical and Health Research involving Human Subjects by the Ministry of Education, Culture, Sports, Science and Technology, and the Ministry of Health, Labour and Welfare of Japan.

\section{Clinical assessment}

Clinical assessment involved all courses of MEC in each patient. The control of CINV was evaluated by the rates of no nausea (no episode), no vomiting (no episode), no nausea and vomiting, no rescue (defined as no use of rescue medication), and complete response (defined as no vomiting and no use of rescue medication) in the overall (0-120 hours), acute phase (within 24 hours), and delayed phase (24-120 hours) after chemotherapy during the study period. We also evaluated the surrogate endpoint to compare the proportion of patients who received salvage antiemetic therapy as well as courses that included salvage antiemetic therapy, necessary to control for CINV in both groups during the study period. Furthermore, the integrated costs of antiemetics per course in both groups were evaluated, according to 2014 Japanese medication prices. The total costs per course in the control group and the study group were 152 USD and 132 USD, respectively (1 USD = 101 JPY [June 27, 2016]), while the total cost per course of salvage antiemetic therapy (three-drug combination of aprepitant, palonosetron, and dexamethasone) was 268 USD. In addition, adverse events induced by chemotherapy were also evaluated according to the Common Terminology Criteria for Adverse Events, version 4.0 [10].

\section{Statistical analysis}

Fisher's exact test, $\chi^{2}$ test, and the Mann-Whitney's $U$-test were used to compare the categorical data and continuous data between both groups, as appropriate. The integrated costs of antiemetics per course and chemotherapy dose were indicated as the mean \pm standard deviation. Univariate and multivariate logistic regression analysis was used to identify risk factors associated with salvage antiemetic therapy during all courses. All possible explanatory variables reported by several previous studies [11, 12] were included in the univariate model as independent variables, including the patient's age ( $<55$ or $\geq 55$ years old), sex (female or male), disease (colorectal cancer vs gastric cancer), chemotherapy regimen (oxaliplatin or irinotecan), previous chemotherapy (yes or no), and antiemetic use (three-drug combination or two-drug combination). The possible explanatory variables with $p<0.05$ in univariate analysis were included in the multivariate model. All analyses were performed using JMP 8.0 (SAS Institute, Tokyo, Japan). All $p$ values were two-tailed, and $p<0.05$ was considered significant.

\section{Results}

In total, 443 treatment courses were evaluated in 83 patients. Baseline patient characteristics are summarized in Table 1 . The number of oxaliplatin-based regimens in the control group and the study group was $40(67.8 \%)$ and $19(79.2 \%)$, respectively. The number of patients who received prior chemotherapy in the control group and the study group was $27(45.8 \%)$ and 5 (20.8\%), respectively, and was statistically significant $(p=$ 0.047).

Table 1. Baseline patient characteristics

\begin{tabular}{|c|c|c|c|c|}
\hline & & $\begin{array}{l}\text { Control group } \\
(\mathrm{n}=59)\end{array}$ & $\begin{array}{l}\text { Study group } \\
(\mathrm{n}=24)\end{array}$ & $p$ value \\
\hline \multirow[t]{3}{*}{ Age (years) } & Median (range) & $65(32-86)$ & $66(50-77)$ & 0.932 \\
\hline & $\geq 55, \mathrm{n}(\%)$ & $55(93.2)$ & $22(91.7)$ & 1.000 \\
\hline & $<55, \mathrm{n}(\%)$ & $4(6.8)$ & $2(8.3)$ & \\
\hline \multirow[t]{2}{*}{ Sex } & Male, n (\%) & $31(52.5)$ & $18(75.0)$ & 0.085 \\
\hline & Female, n (\%) & $28(47.5)$ & $6(25.0)$ & \\
\hline \multirow[t]{2}{*}{ Disease } & Colorectal, n (\%) & $51(86.4)$ & $22(91.7)$ & 0.716 \\
\hline & Gastric, n (\%) & 8 (13.6) & $2(8.3)$ & \\
\hline \multirow[t]{2}{*}{$\begin{array}{l}\text { Chemotherapy } \\
\text { regimen }\end{array}$} & $\begin{array}{l}\text { Oxaliplatin, n } \\
(\%)\end{array}$ & $40(67.8)$ & $19(79.2)$ & 0.424 \\
\hline & Irinotecan, $\mathrm{n}(\%)$ & $19(32.2)$ & $5(20.8)$ & \\
\hline $\begin{array}{l}\text { Oxaliplatin dose } \\
\left(\mathrm{mg} / \mathrm{m}^{2}\right)\end{array}$ & Median (range) & $100(63-130)$ & $83(75-130)$ & 0.910 \\
\hline $\begin{array}{l}\text { Irinotecan dose } \\
\left(\mathrm{mg} / \mathrm{m}^{2}\right)\end{array}$ & Median (range) & $144(75-150)$ & $142(139-150)$ & 0.413 \\
\hline \multirow{2}{*}{$\begin{array}{l}\text { Prior } \\
\text { chemotherapy }\end{array}$} & Yes, n (\%) & $27(45.8)$ & $5(20.8)$ & $0.047^{*}$ \\
\hline & No, n (\%) & $32(54.2)$ & $19(79.2)$ & \\
\hline
\end{tabular}

Fisher's exact test and Mann-Whitney's $U$-test were used to compare the categorical data and continuous data between both groups, respectively. ${ }^{*} p<0.05$

Comparison of the true endpoint of CINV between all courses and salvage courses in both groups is shown in Table 2. The study group had significantly higher percentages of no rescue and 
complete response than the control group in the overall, acute phase, and delayed phase during all courses, whereas the control group had significantly higher percentages of no nausea and no nausea and vomiting than the study group in the overall, acute phase, and delayed phase during all courses. On the other hand, the rates of severe nausea (grade $\geq 2$ ) in the control group during all courses were slightly higher than the study group $(9.0 \%, 3.6 \%$, and $8.7 \%$ versus $4.5 \%, 2.7 \%$, and $4.5 \%$ in the overall, acute phase, and delayed phase, respectively, which was not statistically significant). In addition, the proportion of patients who had severe nausea (grade $\geq 2$ ) in the control group and study group in the overall during all courses was $28.8 \%(17 / 59)$ and 16.7 $\%(4 / 24)$, respectively, and was not statistically significant $(p=0.282)$.

Table 2. Comparison of proportion of efficacy parameter of CINV between all courses and salvage courses in both groups

\begin{tabular}{|c|c|c|c|c|c|c|}
\hline & \multicolumn{3}{|l|}{ All courses } & \multicolumn{3}{|c|}{ Salvage courses } \\
\hline & $\begin{array}{l}\text { Control } \\
\text { group } \\
(\mathrm{n}=59)(\%)\end{array}$ & $\begin{array}{l}\text { Study } \\
\text { group } \\
(\mathrm{n}=24) \\
(\%)\end{array}$ & $p$ value & $\begin{array}{l}\text { Control } \\
\text { group } \\
(\mathrm{n}=19) \\
(\%)\end{array}$ & $\begin{array}{l}\text { Study } \\
\text { group } \\
(\mathrm{n}=2) \\
(\%)\end{array}$ & $\begin{array}{l}p \\
\text { value }\end{array}$ \\
\hline \multicolumn{7}{|l|}{ No nausea } \\
\hline Overall & 82.0 & 70.0 & 0.007 * & 81.9 & 77.8 & 1.000 \\
\hline Acute phase & 93.4 & 82.7 & $<0.001^{*}$ & 90.5 & 77.8 & 1.000 \\
\hline $\begin{array}{l}\text { Delayed } \\
\text { phase }\end{array}$ & 83.2 & 70.9 & $0.005^{*}$ & 81.9 & 88.9 & 1.000 \\
\hline \multicolumn{7}{|l|}{ No vomiting } \\
\hline Overall & 95.2 & 98.2 & 0.169 & 94.0 & 100 & 1.000 \\
\hline Acute phase & 99.4 & 100 & 0.415 & 99.1 & 100 & 1.000 \\
\hline $\begin{array}{l}\text { Delayed } \\
\text { phase }\end{array}$ & 95.5 & 98.2 & 0.204 & 94.0 & 100 & 1.000 \\
\hline \multicolumn{7}{|c|}{ No nausea and vomiting } \\
\hline Overall & 82.0 & 70.0 & $0.007^{*}$ & 81.9 & 77.8 & 1.000 \\
\hline Acute phase & 93.4 & 82.7 & $<0.001^{*}$ & 90.5 & 77.8 & 1.000 \\
\hline $\begin{array}{l}\text { Delayed } \\
\text { phase }\end{array}$ & 83.2 & 70.9 & $0.005^{*}$ & 81.9 & 88.9 & 1.000 \\
\hline \multicolumn{7}{|l|}{ No rescue } \\
\hline Overall & 50.8 & 64.5 & $0.012^{*}$ & 55.2 & 88.9 & 0.077 \\
\hline Acute phase & 51.1 & 65.5 & $0.009^{*}$ & 55.2 & 88.9 & 0.077 \\
\hline $\begin{array}{l}\text { Delayed } \\
\text { phase }\end{array}$ & 50.8 & 64.5 & $0.012^{*}$ & 55.2 & 88.9 & 0.077 \\
\hline \multicolumn{7}{|l|}{$\begin{array}{l}\text { Complete } \\
\text { response }\end{array}$} \\
\hline Overall & 50.2 & 63.6 & $0.014^{*}$ & 55.2 & 88.9 & 0.077 \\
\hline Acute phase & 51.1 & 65.5 & $0.009^{*}$ & 55.2 & 88.9 & 0.077 \\
\hline $\begin{array}{l}\text { Delayed } \\
\text { phase }\end{array}$ & 50.2 & 63.6 & $0.014^{*}$ & 55.2 & 88.9 & 0.077 \\
\hline
\end{tabular}

Metoclopramide and domperidone, a dopamine receptor antagonist, was used 36 patients and 8 patients, respectively, as a rescue medication to control sudden episodes of CINV in both groups. In addition, oxaliplatin dose in the control group and the study group during all courses was $94 \pm 23 \mathrm{mg} / \mathrm{m}^{2}$ and $95 \pm 23 \mathrm{mg} / \mathrm{m}^{2}$, respectively, and was not statistically significant $(p=0.429)$, whereas irinotecan dose in the control group and the study group during all courses was $126 \pm 20 \mathrm{mg} / \mathrm{m}^{2}$ and $143 \pm 8 \mathrm{mg} / \mathrm{m}^{2}$, respectively, and was statistically significant $(p<$ 0.0001).

Subsequently, we compared the proportion of patients who received salvage antiemetic therapy as well as courses that included salvage antiemetic therapy between both groups during the study period. The proportion of patients and courses that included salvage antiemetic therapy in both groups is shown in Fig. 2. The proportion of patients who received salvage antiemetic therapy in the control group and the study group was $32.2 \%(19 / 59)$ and 8.3 $\%(2 / 24)$, respectively, and was statistically significant $(p=0.027)$. Furthermore, the proportion of courses which included salvage antiemetic therapy in the control group and the study group was $34.8 \%$ $(116 / 333)$ and $8.2 \%(9 / 110)$, respectively, and was also statistically significant $(p<0.001)$. The mean integrated costs of antiemetics per course in the control group and the study group were $193 \pm 55$ USD and $143 \pm 38$ USD, respectively. The difference between the control group and the study group was mostly dependent on the proportion that received an expensive salvage antiemetic therapy, administered as modified antiemetics for breakthrough patients.

The results of the univariate and multivariate logistic regression analysis to identify risk factors associated with salvage antiemetic therapy during all courses are shown in Fig. 3. Univariate logistic regression analysis revealed that female patients were significantly associated with an increased risk of salvage antiemetic therapy (crude odds ratio [OR]: $3.02,95 \%$ confidence interval [CI]: 1.10-8.59; $p=$ 0.032), whereas a three-drug combination was significantly associated with a reduced risk of salvage antiemetic therapy (crude OR: 0.19, 95 \% CI: 0.03-0.74; $p=0.015$ ). Multivariate logistic regression analysis also revealed that a three-drug combination was significantly associated with a reduced risk of salvage antiemetic therapy (adjusted OR: 0.23, 95 \% CI: $0.03-0.93 ; p=0.038$ ), whereas female patients was not significantly associated with an increased risk of salvage antiemetic therapy (adjusted OR: 2.49, $95 \%$ CI: $0.88-7.27 ; p=0.087$ ).

\section{Discussion}

There is no information available at present regarding the therapeutic benefit of a three-drug combination of a $\mathrm{NK}_{1} \mathrm{RA}, 5-\mathrm{HT}_{3} \mathrm{RA}$, and dexamethasone in gastrointestinal cancer patients who received MEC in a clinical practice setting. The present study shows that antiemetic effectiveness and cost-saving of a three-drug combination of aprepitant, 
generic granisetron, and dexamethasone was superior to that of a two-drug combination of palonosetron plus dexamethasone. We also used a multivariate logistic regression analysis to demonstrate, for the first time, that a three-drug combination was significantly associated with a reduced risk of salvage antiemetic therapy.
(A)

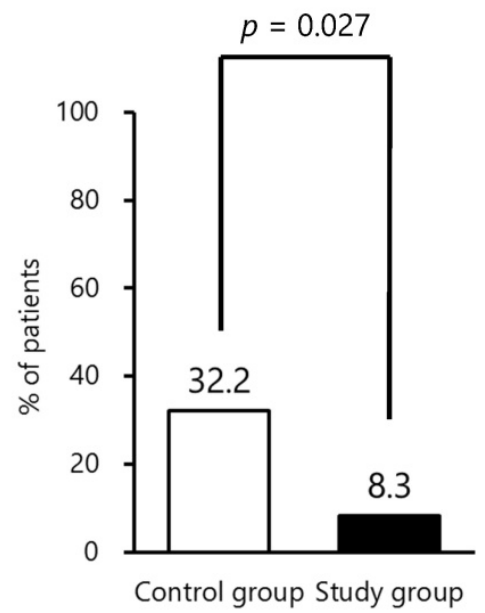

(B)

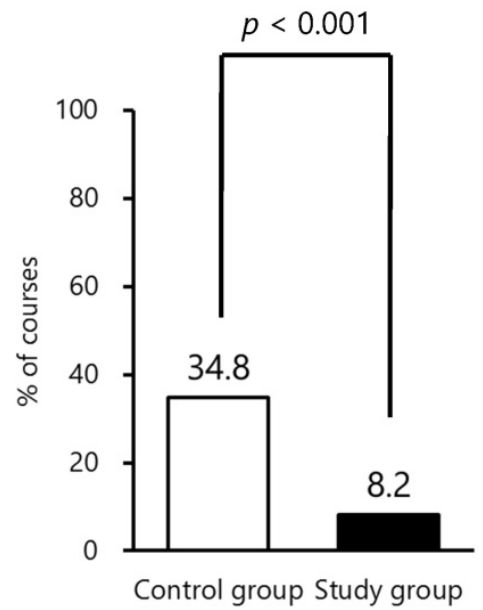

Figure 2. The proportion of patients and courses that included salvage antiemetic therapy in both groups. (A) The proportion of patients, (B) The proportion of courses. Fisher's exact test was used to compare the categorical data between both groups.

(A)

\begin{tabular}{|c|c|c|c|c|c|c|}
\hline Variables & & Case / Control Less & More & Crude OR & $95 \% \mathrm{Cl}$ & $p$ value \\
\hline \multirow[t]{2}{*}{ Age (years) } & $<55$ & $2 / 4$ & & 1.53 & $(0.20-8.48)$ & 0.648 \\
\hline & $\geq 55$ & $19 / 58$ & & 1.00 & & \\
\hline \multirow[t]{2}{*}{ Sex } & Female & $12 / 19$ & & 3.02 & $(1.10-8.59)$ & $0.032^{*}$ \\
\hline & Male & $9 / 43$ & & 1.00 & & \\
\hline \multirow[t]{2}{*}{ Disease } & Colorectal & $19 / 54$ & & 1.41 & $(0.32-9.87)$ & 0.674 \\
\hline & Gastric & $2 / 8$ & & 1.00 & & \\
\hline \multirow[t]{2}{*}{ Chemotherapy regimen } & Oxaliplatin & $13 / 46$ & - & 0.57 & $(0.20-1.65)$ & 0.291 \\
\hline & Irinotecan & $8 / 16$ & & 1.00 & & \\
\hline \multirow[t]{2}{*}{ Prior chemotherapy } & Yes & $11 / 21$ & & 2.15 & $(0.79-5.97)$ & 0.136 \\
\hline & No & $10 / 41$ & & 1.00 & & \\
\hline \multirow[t]{4}{*}{ Antiemetics } & Three-drug & $2 / 22$ & & 0.19 & $(0.03-0.74)$ & $0.015^{*}$ \\
\hline & Two-drug & $19 / 40$ & & 1.00 & & \\
\hline & & 0.1 & 1 & 0 & & \\
\hline & \multicolumn{6}{|c|}{ Crude OR $(95 \% \mathrm{Cl})$} \\
\hline
\end{tabular}

(B)

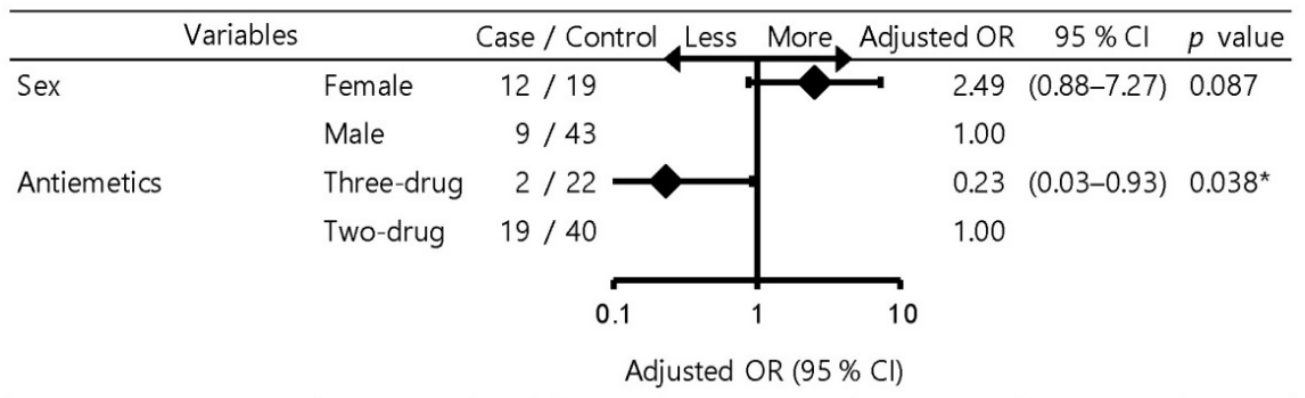

Figure 3. Forest plot of univariate and multivariate analysis for risk factors associated with salvage antiemetic therapy. (A) Univariate analysis, (B) Multivariate analysis. OR: odds ratio; $\mathrm{Cl}$ : confidence interval. Logistic regression analysis was used to identify the risk factors associated with salvage antiemetic therapy during all courses. $* p<0.05$ 
The present study demonstrates that the true endpoint and the surrogate endpoint of a three-drug combination was significantly superior to that of a two-drug combination. To our knowledge, the therapeutic benefit of a three-drug combination of a $\mathrm{NK}_{1} \mathrm{RA}, 5-\mathrm{HT}_{3} \mathrm{RA}$, and dexamethasone has not previously been established for MEC [5-8]. A randomized, placebo-controlled phase III trial showed that a three-drug combination was superior to a two-drug combination in autologous transplantation patients who had received high-dose melphalan [13]. Schmitt et al. [13] reported in 362 patients receiving autologous transplantation even though MEC, but not outpatient chemotherapy setting. That was far from in the present study setting. On the other hand, a recent phase III trial also showed that a three-drug combination was superior to a two-drug combination for colorectal cancer patients who had received an oxaliplatin-based regimen [9]. In addition, a three-drug combination had almost comparable antiemetic efficacy between a first- and second-generation 5- $\mathrm{HT}_{3}$ RA [9]. Our data support these findings. In this study, we considered the antiemetic effectiveness and cost-saving between a two- and three-drug combination by using the difference between a first- and second-generation $5-\mathrm{HT}_{3} \mathrm{RA}$, even when adding the aprepitant. Previous trials showed the superiority of a three-drug combination for MEC [9, 13], but a three-drug combination is generally more expensive than a two-drug combination. The novelty of our own findings was the antiemetic effectiveness as well as cost-saving of a three-drug combination. In Japan, granisetron is available as a generic drug. Therefore, the total cost per course of a three-drug combination of aprepitant, generic granisetron, and dexamethasone was less than that of a two-drug combination of palonosetron plus dexamethasone, even when adding the aprepitant. Taken together, a three-drug combination of a $\mathrm{NK}_{1} \mathrm{RA}, 5-\mathrm{HT}_{3} \mathrm{RA}$, and dexamethasone has a superior therapeutic benefit for MEC than a two-drug combination of a 5- $\mathrm{HT}_{3}$ RA plus dexamethasone. Furthermore, the use of a first-generation $5-\mathrm{HT}_{3} \mathrm{RA}$, especially a generic drug, should be strongly considered in a three-drug combination to help reducing health care costs. Our findings suggest that the antiemetic effectiveness and cost-saving of a three-drug combination of aprepitant, generic granisetron, and dexamethasone was superior to that of a two-drug combination of palonosetron plus dexamethasone for gastrointestinal cancer patients who received MEC.

In the present study, the rates of no vomiting in the acute phase and delayed phase in the study group during all courses was $100 \%$ and $98.2 \%$, respectively.
Our data on the control of vomiting were generally consistent with SENRI trial [9], which showed in 413 patients receiving oxaliplatin-based regimen that the rates of no vomiting in the acute phase and delayed phase in three-drug combination group was $100 \%$ and $95.7 \%$, respectively. On the other hand, the complete response ratio in the acute phase and delayed phase in the study group during all courses was $65.5 \%$ and $63.6 \%$, respectively. Our data on the complete response ratio were inconsistent with SENRI trial [9], which showed that complete response ratio in the acute phase and delayed phase in three-drug combination group was $94.7 \%$ and $85.0 \%$, respectively. The much lower rates of complete response observed in the present study as compared with SENRI trial may be due to a clinical practice setting, but not a prospective clinical trial. Our outpatients were prescribed on-demand use of rescue medication in most cases, therefore, they may be easy to use rescue medication rather than those in SENRI trial.

In the present study, the rates of no nausea and no nausea and vomiting in the overall, acute phase, and delayed phase in the control group during all courses were significantly higher than the study group. However, the rates of severe nausea (grade $\geq 2$ ) in the overall, acute phase, and delayed phase in the control group during all courses were slightly higher than the study group. Therefore, clinicians would decide to modify subsequent courses as a salvage antiemetic therapy in the control group rather than the study group.

As the surrogate endpoint of our clinical assessment, we used the proportion of patients who received salvage antiemetic therapy as well as courses that included salvage antiemetic therapy necessary to control CINV in both groups during the study period. In general, previous phase III trials of antiemetics use a complete response ratio in the overall phase or delayed phase after the first course for each patient as a true endpoint [14, 15]. In our opinion, however, there is no rationale to speculate that the present surrogate endpoint is not an appropriate assessment for antiemetic efficacy, as pharmacists reviewed all outpatients to assess CINV and other adverse events induced by chemotherapy, and consulted physicians to modify salvage antiemetic therapy as needed. In this study, two patients who switched the standard antiemetics from the control group to the study group were subsequently given salvage antiemetic therapy because one patient developed grade 3 nausea and anorexia in the delayed phase and the other patient developed grade 2 nausea and anorexia in the acute and delayed phases. Consequently, no patients discontinued chemotherapy because of CINV in this 
study. Palonosetron, a second-generation $5-\mathrm{HT}_{3} \mathrm{RA}$, has a longer half-life than other first-generation $5-\mathrm{HT}_{3}$ RAs and is also effective against delayed phase CINV for highly emetogenic chemotherapy [14, 15]. In Japan, palonosetron dosing is approved at $0.75 \mathrm{mg}$, 3 -fold higher than in other countries. Taken together, palonosetron, an alternative choice for a first-generation $5-\mathrm{HT}_{3} \mathrm{RA}$, might be an option as a salvage antiemetic therapy, especially for control during the delayed phase.

Multinational Association for Supportive Care in Cancer/European Society for Medical Oncology antiemetic guidelines were updated in March 2016 [5]. The guidelines recommend that the standard antiemetics for CINV in patients receiving carboplatin-based MEC and non-carboplatin MEC should be a three-drug combination of a $\mathrm{NK}_{1} \mathrm{RA}$, $5-\mathrm{HT}_{3} \mathrm{RA}$, and dexamethasone and a two-drug combination of a 5- $\mathrm{HT}_{3}$ RA and dexamethasone, respectively. However, National Comprehensive Cancer Network, American Society of Clinical Oncology, and national antiemetic guidelines recommend different combinations [6, 7, 8]. This conflicting information may be attributable to differences in opinion among committee members, countries, evaluation methods, and approved dose of palonosetron $(0.25 \mathrm{mg}$ in other countries vs. $0.75 \mathrm{mg}$ in Japan).

The present study had several limitations, the first of which was its retrospective, single-institution study design with a small sample size. Our multivariate model included only two variables except for the other clinical important explanatory variables which were reported by several previous studies [11, 12]. That model was based on 83 patients. As a result, the increase of the analytic number was desirable. Second, we could not fully assess the patients' background, including performance status of patients, smoking history, habitual alcohol consumption, motion sickness history, and patients' quality of life as a result of the retrospective nature of the study an outpatient setting, meaning that a degree of bias may have been introduced into our results. Third, we did not define the protocol of study design to modify subsequent courses as a salvage antiemetic therapy because of clinical practice setting. Large-scale, multicenter studies are therefore needed to confirm the findings of our study.

In conclusion, this study is the first to demonstrate that the antiemetic effectiveness and cost-saving of a three-drug combination of aprepitant, generic granisetron, and dexamethasone was superior to that of a two-drug combination of palonosetron plus dexamethasone in gastrointestinal cancer patients who received MEC.

\section{Acknowledgments}

We thank all patients and medical staff at Ehime University Hospital who were involved in this study.

\section{Competing Interests}

The authors have declared that no competing interest exists.

\section{References}

1. Yamada Y, Higuchi K, Nishikawa K, et al. Phase III study comparing oxaliplatin plus S-1 with cisplatin plus S-1 in chemotherapy-naïve patients with advanced gastric cancer. Ann Oncol. 2015; 26: 141-8.

2. Cassidy J, Clarke S, Díaz-Rubio E, et al. Randomized phase III study of capecitabine plus oxaliplatin compared with fluorouracil/folinic acid plus oxaliplatin as first-line therapy for metastatic colorectal cancer. J Clin Oncol. 2008; 26: 2006-12.

3. Hironaka S, Ueda S, Yasui H, et al. Randomized, open-label, phase III study comparing irinotecan with paclitaxel in patients with advanced gastric cancer without severe peritoneal metastasis after failure of prior combination chemotherapy using fluoropyrimidine plus platinum: WJOG 4007 trial. J Clin Oncol. 2013; 31: 4438-44.

4. Muro K, Boku N, Shimada Y, et al. Irinotecan plus S-1 (IRIS) versus fluorouracil and folinic acid plus irinotecan (FOLFIRI) as second-line chemotherapy for metastatic colorectal cancer: a randomised phase $2 / 3$ non-inferiority study (FIRIS study). Lancet Oncol. 2010; 11: 853-60.

5. [Internet] Multinational Association for Supportive Care in Cancer/European Society for Medical Oncology (MASCC/ESMO). Antiemetic Guidelines Version 1.1 - Updated March 2016. http://www.mascc.org/ antiemetic-guidelines.

6. [Internet] National Comprehensive Cancer Network (NCCN). Clinical Practice Guidelines in Oncology (NCCN Guidelines ${ }^{\circledR}$ ) Antiemesis; Version 2. 2016. https://www.nccn.org/professionals/physician_gls/pdf/antiemesis. pdf.

7. Hesketh PJ, Bohlke K, Lyman GH, et al. Antiemetics: American Society of Clinical Oncology Focused Guideline Update. J Clin Oncol. 2016; 34: 381-6.

8. Takeuchi H, Saeki T, Aiba K, et al. Japanese Society of Clinical Oncology clinical practice guidelines 2010 for antiemesis in oncology: executive summary. Int J Clin Oncol. 2016; 21: 1-12.

9. Nishimura J, Satoh T, Fukunaga M, et al. Combination antiemetic therapy with aprepitant/fosaprepitant in patients with colorectal cancer receiving oxaliplatin-based chemotherapy (SENRI trial): a multicentre, randomised, controlled phase 3 trial. Eur J Cancer. 2015; 51: 1274-82.

10. [Internet] Common Terminology Criteria for Adverse Events. http://evs.nci.nih.gov/ftp1/CTCAE/About.html.

11. Sekine I, Segawa Y, Kubota K, et al. Risk factors of chemotherapy-induced nausea and vomiting: index for personalized antiemetic prophylaxis. Cancer Sci. 2013; 104: 711-7.

12. Tamura K, Aiba K, Saeki T, et al. Testing the effectiveness of antiemetic guidelines: results of a prospective registry by the CINV Study Group of Japan. Int J Clin Oncol. 2015; 20: 855-65.

13. Schmitt T, Goldschmidt H, Neben K, et al. Aprepitant, granisetron, and dexamethasone for prevention of chemotherapy-induced nausea and vomiting after high-dose melphalan in autologous transplantation for multiple myeloma: results of a randomized, placebo-controlled phase III trial. J Clin Oncol. 2014; 32: 3413-20.

14. Suzuki K, Yamanaka T, Hashimoto H, et al. Randomized, double-blind, phase III trial of palonosetron versus granisetron in the triplet regimen for preventing chemotherapy-induced nausea and vomiting after highly emetogenic chemotherapy: TRIPLE study. Ann Oncol. 2016; 27: 1601-6.

15. Saito M, Aogi K, Sekine I, et al. Palonosetron plus dexamethasone versus granisetron plus dexamethasone for prevention of nausea and vomiting during chemotherapy: a double-blind, double-dummy, randomised, comparative phase III trial. Lancet Oncol. 2009; 10: 115-24. 